
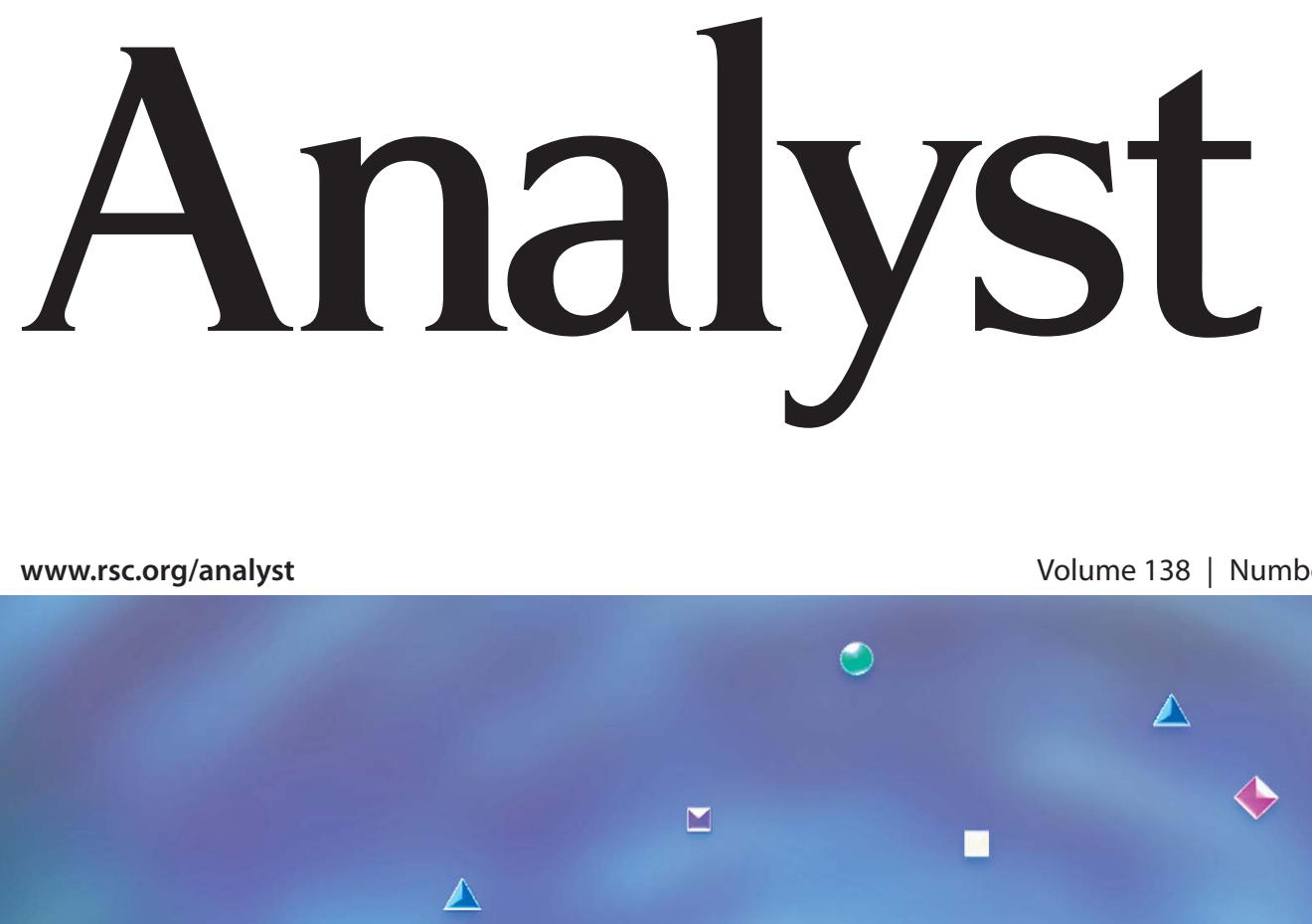

$\triangle$

$\odot$

$\odot$

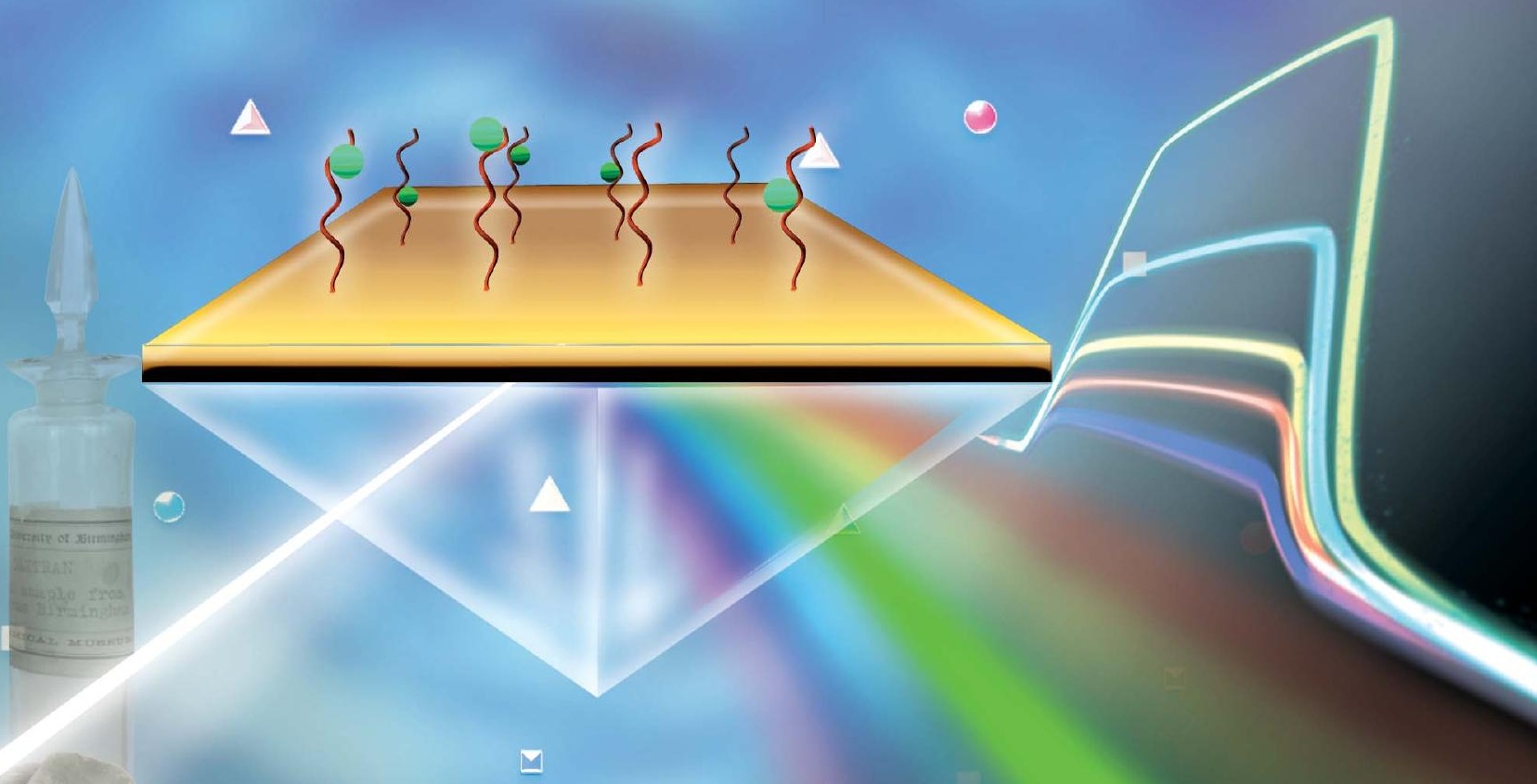




\section{Glucose selective Surface Plasmon Resonance-based bis-boronic acid sensort:}

Cite this: Analyst, 2013, 138, 7140

\author{
Alex Stephenson-Brown, ${ }^{a}$ Hui-Chen Wang, ${ }^{\text {bc }}$ Parvez Iqbal, ${ }^{a}$ Jon A. Preece, ${ }^{c}$ \\ Yitao Long, ${ }^{d}$ John S. Fossey, ${ }^{* c}$ Tony D. James ${ }^{\star b}$ and Paula M. Mendes ${ }^{\star a}$
}

Saccharides - a versatile class of biologically important molecules - are involved in a variety of physiological and pathological processes, but their detection and quantification is challenging. Herein, surface plasmon resonance and self-assembled monolayers on gold generated from bis-boronic acid bearing a thioctic acid moiety, whose intramolecular distance between the boronic acid moieties is well defined, are shown to detect D-glucose with high selectivity, demonstrating a higher affinity than other saccharides probed, namely D-galactose, D-fructose and D-mannose.
Received 29th April 2013

Accepted 18th September 2013

DOI: $10.1039 / c 3 a n 01233 f$

www.rsc.org/analyst several strategies, including electrochemical peroxide measurement where, at a constant voltage, the current generated across the electrochemical cell is proportional to the concentration of hydrogen peroxide, which is in turn proportional to the glucose. ${ }^{7}$ Other methods used have included monitoring changes in mechanical and optical properties of polyelectrolyte gels induced by glucose oxidation, and subsequent changes produced in the gels ionic environment. ${ }^{8}$

Despite their widespread use, enzyme-based sensor systems for glucose often suffer from a number of limitations. Notably, they result in the consumption of the analyte of interest from samples, can be dependent on local oxygen concentrations and, like all proteins, are poorly heat stable and prone to reduced activity over time owing to loss of functional enzyme due to denaturation. ${ }^{9}$ Due to these limitations, there is a drive towards non-protein dependent systems, ${ }^{10}$ which harbour the potential for vast improvements to current glucose monitoring technologies.

One group of compounds which is well suited to this challenge is boronic acids, which are able to readily and reversibly form cyclic boronate esters with diols in aqueous basic media. ${ }^{\mathbf{1 1}}$ Since saccharides contain 1,2- and 1,3-diol units they provide an ideal structural framework for binding to boronic acids. ${ }^{12}$ It is this property which, in recent years, has led to a growing interest in the development of detection and sensor systems that employ boronic acid groups as "synthetic lectins"., $5,7,11 b, 13$ The most favoured class of boronic acid-based sensors utilise an amine group proximal to a phenylboronic acid group, in which the Lewis acid-Lewis base interaction between the boronic acid and the tertiary amine enables the formation of boronate esters to proceed at neutral $\mathrm{pH} .^{7,11 c, 11 d}$ However, interactions between phenylboronic acids and saccharides are generally of limited selectivity and typically they display a high affinity for fructose over other monosaccharides. ${ }^{\mathbf{1 1 f}, \mathbf{1 4}}$

By employing two intramolecular phenylboronic acid receptor units selectivity in favour of glucose may be 
achieved. ${ }^{11 c, 12}$ By using a six carbon linker unit, single molecules of D-glucose were shown to bind to bis-boronic acid (bis-BA) binding motifs using two sets of diols, thus forming stable, cyclic 1:1 complexes with a higher stability than other saccharides such as D-fructose, D-galactose and D-mannose. ${ }^{11 c}$ Although the selective binding of glucose has been demonstrated in solution, surface-based sensing platforms comprising these novel bis-BA derivatives still need to be developed as only then can their potential be fully exploited for highly sensitive, robust, and selective saccharide sensors.

This work forms part of a dual submission, a novel glucose selective receptor, designed for surface functionalisation, was synthesised and subsequently has been employed in a variety of settings. In this paper, a surface plasmon resonance (SPR) detection regime is used to probe the saccharide binding, whilst the partner paper uses electrochemical responses of a bisboronic acid functionalised surface. ${ }^{15}$

With this proviso mind, we report the design and fabrication of a glucose selective surface sensor, using the principle of selfassembly to form the sensor surface and employing SPR optical technique to detect and measure the relative binding of analyte to the surface sensor. With its high sensitivity, and unique capacity for label-free and real time detection of molecular interactions, ${ }^{16}$ SPR provides an attractive sensing platform on which to build systems to monitor analytes, such as glucose, within physiological scenarios. Furthermore, recent developments towards easily transportable miniaturised SPR systems ${ }^{\mathbf{1 7}}$ makes SPR a highly attractive platform for medical sensor applications, particularly with reference to application in near patient testing.

The functional sensing surface was fabricated by formation of a two-component, mixed self-assembled monolayer (SAM) on a gold surface (Fig. 1). One of the components of the SAM is the

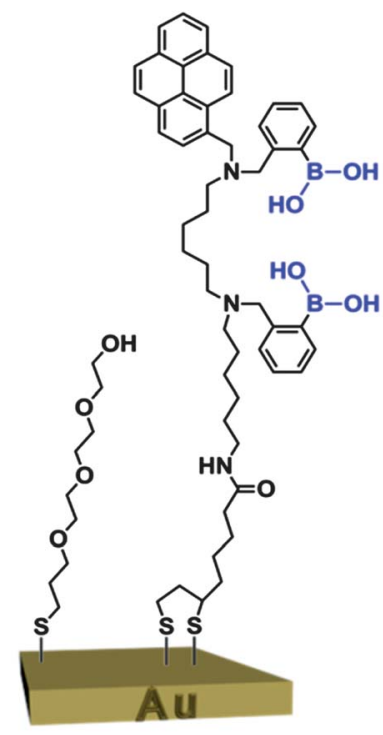

Bis-BA:TEGT SAM

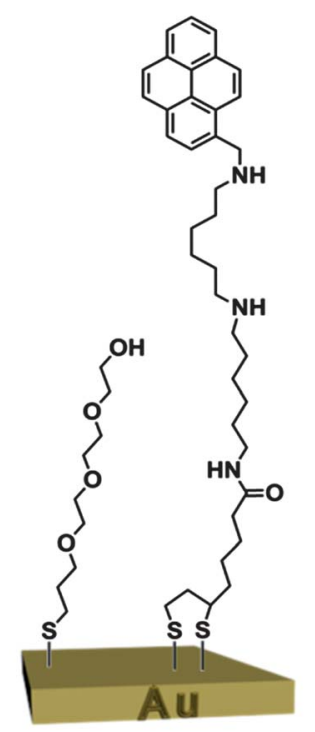

Diamine:TEGT SAM
Fig. 1 Two-component, mixed SAM from a bis-BA derivative and a TEGTterminated thiol. The control two-component mixed SAM, diamine : TEGT SAM, lacks the phenylboronic acid moieties. previously described bis-BA derivative (separated by a six-carbon linker), ${ }^{12}$ which now bears a thioctic acid appended unit for binding to the gold surface. A tri(ethylene glycol)-terminated thiol (TEGT), described previously, ${ }^{\mathbf{1 6} a}$ was used as the second SAM component to ensure adequate separation between adjacent bis-BA on the surface, and eliminate the possibility of neighbouring boronic acid groups inhibiting saccharide binding or selectivity. In complex biological conditions, the presence of the TEGT on the surface could also serve as a shielding component to prevent non-specific protein adsorption. ${ }^{18}$ A control surface was also produced, in the same manner as described above but with a non-active compound (diamine) in place of the bis-BA (Fig. 1). The control molecule was structurally similar to the bis-BA molecule except for the absence of the phenylboronic acid moieties.

\section{Experimental}

\section{SAM preparation}

Polycrystalline gold substrates were purchased from George Albert PVD, Germany and consisted of a $50 \mathrm{~nm}$ gold layer deposited onto a glass covered with a thin layer of chromium. The $\mathrm{Au}$ substrates were cleaned by immersion in piranha solution (7:3, $\mathrm{H}_{2} \mathrm{SO}_{4}: \mathrm{H}_{2} \mathrm{O}_{2}$ ) at room temperature for $10 \mathrm{~min}$. (Caution: Piranha solution reacts violently with all organic compounds and should be handled with care.) Samples removed from the piranha solution were immediately rinsed with Ultra High Pure (UHP) $\mathrm{H}_{2} \mathrm{O}$, followed by HPLC grade methanol (Fischer Scientific) for $1 \mathrm{~min}$. Immediately after cleaning, the substrates were immersed in freshly prepared $0.1 \mathrm{mM}$ methanolic solutions of the either pure or mixed bis-BA, TEGT or diamine compound. Post-immersion in the SAM forming solution, the substrates were rinsed with HPLC $\mathrm{MeOH}$ and dried with a stream of argon. Details of the synthesis of diamine may be found in the ESI. $\uparrow$ Details of synthesis of bis-BA can be found in the accompanying paper. ${ }^{15}$

\section{Contact angle}

Contact angles were determined using a home-built contact angle apparatus, equipped with a charged coupled device (CCD) KP-M1E/K camera (Hitachi) that was attached to a personal computer for video capture. The dynamic contact angles were recorded as a micro-syringe was used to quasi-statically add liquid to or remove liquid from the drop. The drop was shown as a live video image on the PC screen and the acquisition rate was 4 frames per second. FTA Video Analysis software v1.96 (First Ten Angstroms) was used for the analysis of the contact angle of a droplet of UHP $\mathrm{H}_{2} \mathrm{O}$ at the three-phase intersection. The averages and standard errors of contact angles were determined from five different measurements made for each type of SAM.

\section{Ellipsometry}

The thickness of the deposited monolayers was determined by spectroscopic ellipsometry. A Jobin-Yvon UVISEL ellipsometer with a xenon light source was used for the measurements. The 
angle of incidence was fixed at $70^{\circ}$. A wavelength range of $280-$ $820 \mathrm{~nm}$ was used. The DeltaPsi software was employed to determine the thickness values and the calculations were based on a three-phase ambient/SAM/Au model, in which the SAM was assumed to be isotropic and assigned a refractive index of 1.50. The thickness reported is the average and standard error of six measurements taken on each SAM.

\section{X-ray photoelectron spectroscopy (XPS)}

Elemental composition of the SAMs were analysed using an Escalab 250 system (Thermo VG Scientific) operating with Avantage v1.85 software under a pressure of $\sim 5 \times 10^{-9}$ mbar. An $\mathrm{Al} \mathrm{K} \alpha \mathrm{X}$-ray source was used, which provided a monochromatic X-ray beam with incident energy of $1486.68 \mathrm{eV}$. A circular spot size of $\sim 0.2 \mathrm{~mm}^{2}$ was employed. The samples were attached onto a stainless steel holder using double-sided carbon sticky tape (Shintron tape). In order to minimise charge retention on the sample, the samples were clipped onto the holder using stainless steel or $\mathrm{Cu}$ clips. The clips provided a link between the sample and the sample holder for electrons to flow, which the glass substrate inhibits. Low resolution survey spectra were obtained using a pass energy of $150 \mathrm{eV}$ over a binding energy range of $0 \mathrm{eV}$ to $1250 \mathrm{eV}$ obtained using $1 \mathrm{eV}$ increments. The spectra recorded were an average of 3 scans. The high resolution spectra were obtained using a pass energy of $20 \mathrm{eV}$ and $0.1 \mathrm{eV}$ increments over a binding energy range of 20-30 eV, centred on the binding energy of the electron environment being studied. A dwell time of $50 \mathrm{~ms}$ was employed between each binding energy increment. The spectra recorded were an average of between 5 and 250 scans $(\mathrm{N}(1 \mathrm{~s})=100, \mathrm{Au}$ $(4 \mathrm{f})=5, \mathrm{~S}(2 \mathrm{p})=150, \mathrm{~B}(1 \mathrm{~s})=250, \mathrm{O}(1 \mathrm{~s})=50, \mathrm{C}(1 \mathrm{~s})=50)$. Sensitivity factors used in this study were: N (1s), 1.8; Au (4f), 17.1; S (2p), 1.68; B (1s), 0.486; O (1s), 2.93; C (1s), 1.0.

\section{Surface plasmon resonance (SPR)}

SPR experiments were performed with a Reichert SR7000DC Dual Channel Spectrometer (Buffalo, NY, USA) at $25{ }^{\circ} \mathrm{C}$. Prior to the saccharide binding studies, a baseline for the SAMs was established by running degassed PBS pH 7.4 through the machine at a flow rate of $25 \mu \mathrm{l} \mathrm{min}^{-1}$. The SAMs were subsequently exposed to solutions of monosaccharides (0.6 mM, 1.25 mM, $2.5 \mathrm{mM}, 5 \mathrm{mM}$, $10 \mathrm{mM}$ and $20 \mathrm{mM}$ ) in PBS injected at $25 \mu \mathrm{l} \mathrm{min}^{-1}$ for $5 \mathrm{~min}$, after which PBS was again injected over the surface.

\section{Results and discussion}

\section{SAM characterisation}

The advancing contact angle for bis-BA SAM was in good agreement with the literature for pyrene-terminated monolayers, ${ }^{19}$ noting that the hysteresis $\left(\theta_{\mathrm{Adv}}-\theta_{\mathrm{Rec}}\right)$ value of $7^{\circ}$ suggests the presence of a packed monolayer. For the diamine molecule, similar results were observed, however there was a small reduction in the hydrophobicity, which is likely due to the loss of the phenyl groups. As expected, the TEGT molecule formed a hydrophilic monolayer with a hysteresis of $3^{\circ}$. The ellipsometric thickness of the pure formed SAMs, is less than the theoretical molecular length of the molecules (Table 1). This discrepancy, between molecular length and SAM thickness, is expected, in agreement with the literature, and it is ascribed to both the tilt angle and density of the SAM surfactants. ${ }^{20}$

Following contact angle and ellipsometric characterisation of the pure SAMs, studies were conducted to optimise a bisBA : TEGT SAM ratio of $1: 1$ on the gold surface. This optimum ratio should enable maximum sugar binding capacity while avoiding steric hindrance from neighbouring bis-BA molecules in the SAM. As reported in previous literature, ${ }^{\mathbf{1 4 2 1}}$ when producing mixed SAMs, the ratio of two-components in solution are rarely identical to those observed in the SAM, due to the preferential adsorption of one of the components. Thus, systematic studies were carried out in order to understand how the ratios of SAM components in solution diverge from the ratios in the formed SAM. A simple method of quantifying this was to use the relationship proposed by Cassie ${ }^{22}$ which relates the contact angle of a surface of mixed composition to those of pure SAMs (eqn (1)).

$$
\cos \theta_{\mathrm{Adv}}=x \cos \theta_{\mathrm{Adv} 1}+y \cos \theta_{\mathrm{Adv} 2}
$$

Where $\theta_{\mathrm{Adv}}$ is the water advancing contact angle on the mixed $\mathrm{SAM}, \theta_{\mathrm{Adv} 1}$ and $\theta_{\mathrm{Adv} 2}$ are the contact angles related to the pure SAMs formed from bis-BA and TEGT, respectively, and $x$ and $y$ are their corresponding surface molar ratios $(x+y=1)$. Mixed SAMs of different solution concentration ratios of bis-BA and TEGT (1:1, $1: 5,1: 10$ and $1: 40)$ were formed for 24 hours and analysed by contact angle. Using eqn (1), molar ratios (bisBA : TEGT) in the SAMs of $3: 1,3: 2,1: 1$ and $2: 3$ were obtained, respectively. These results indicated that in order to produce a 1:1 bis-BA: TEGT SAM a solution ratio of

Table 1 Advancing and receding water contact angles and ellipsometric thickness values for the different SAMs formed for $24 \mathrm{~h}$

\begin{tabular}{|c|c|c|c|c|}
\hline \multirow[b]{2}{*}{ SAM } & \multicolumn{2}{|c|}{ Contact angle $\left(^{\circ}\right)$} & \multicolumn{2}{|c|}{ Thickness (nm) } \\
\hline & Advancing & Receding & Theoretical $^{a}$ & Experimental \\
\hline Bis-BA & $90.0 \pm 2.5$ & $83.3 \pm 5.6$ & 3.61 & $2.12 \pm 0.12$ \\
\hline Diamine & $85.4 \pm 2.1$ & $79.1 \pm 3.2$ & 3.61 & $2.35 \pm 0.23$ \\
\hline TEGT & $50.3 \pm 1.8$ & $47.1 \pm 2.1$ & 1.66 & $1.17 \pm 0.11$ \\
\hline $1: 1$ bis-BA : TEGT & $77.8 \pm 1.5$ & $64.2 \pm 3.9$ & - & $2.17 \pm 0.35$ \\
\hline $1: 1$ diamine $:$ TEGT & $69.4 \pm 0.7$ & $60.4 \pm 3.0$ & - & $2.10 \pm 0.34$ \\
\hline
\end{tabular}

${ }^{a}$ Theoretical thickness was determined using ChemBio 3D Ultra 11.0. 
components in a 1:10 ratio (bis-BA: TEGT) must be used. Contact angle data from the 1:10 mixed monolayers of the diamine : TEGT SAMs were used to verify the results for the control surfaces, and found to be in good agreement.

As shown in Table 1, and consistent with a mixed monolayer, the $1: 1$ bis-BA : TEGT and $1: 1$ diamine : TEGT SAMs exhibited contact angle and thickness values between those of the pure monolayers. Furthermore, the heterogeneity of the surface due to the presence of both molecules, either bis-BA and TEGT or diamine and TEGT, has led to a greater contact angle hysteresis on the $1: 1$ mixed surfaces than on the pure monolayers.

XPS confirmed the formation of pure and mixed SAMs, showing signals from C (1s), O (1s) and S (2p). High-resolution scans of the $\mathrm{N}(1 \mathrm{~s})$ and $\mathrm{B}(1 \mathrm{~s})$ regions (Fig. 2) show the presence of nitrogen and boron on the pure bis-BA SAMs and bisBA : TEGT mixed SAMs, whereas, as expected, no boron peaks were observed in the mixed diamine: TEGT SAMs. XPS also confirmed the absence of nitrogen and boron on the pure TEGT SAM. For both a pure bis-BA SAM and bis-BA : TEGT mixed SAM, the $\mathrm{B}(1 \mathrm{~s})$ spectra display a peak at $192 \mathrm{eV}$, in good agreement with the values reported for other boronic acid derivatives. ${ }^{23}$ The $\mathrm{N}$ (1s) spectra can be deconvoluted into two peaks, the first one, centred at $400.2 \mathrm{eV}$, is characteristic of amide and amine moieties, while the second peak, centered at $402.0 \mathrm{eV}$, is attributed to protonated amino groups. ${ }^{16 a, 24}$ This finding is not surprising given the structure of the bis-BA molecule; previous studies have observed that the pyrene group is able to promote the protonation of the adjacent nitrogen groups. ${ }^{25}$ In addition, the mildly acidic nature of the methanol used as a SAM solvent
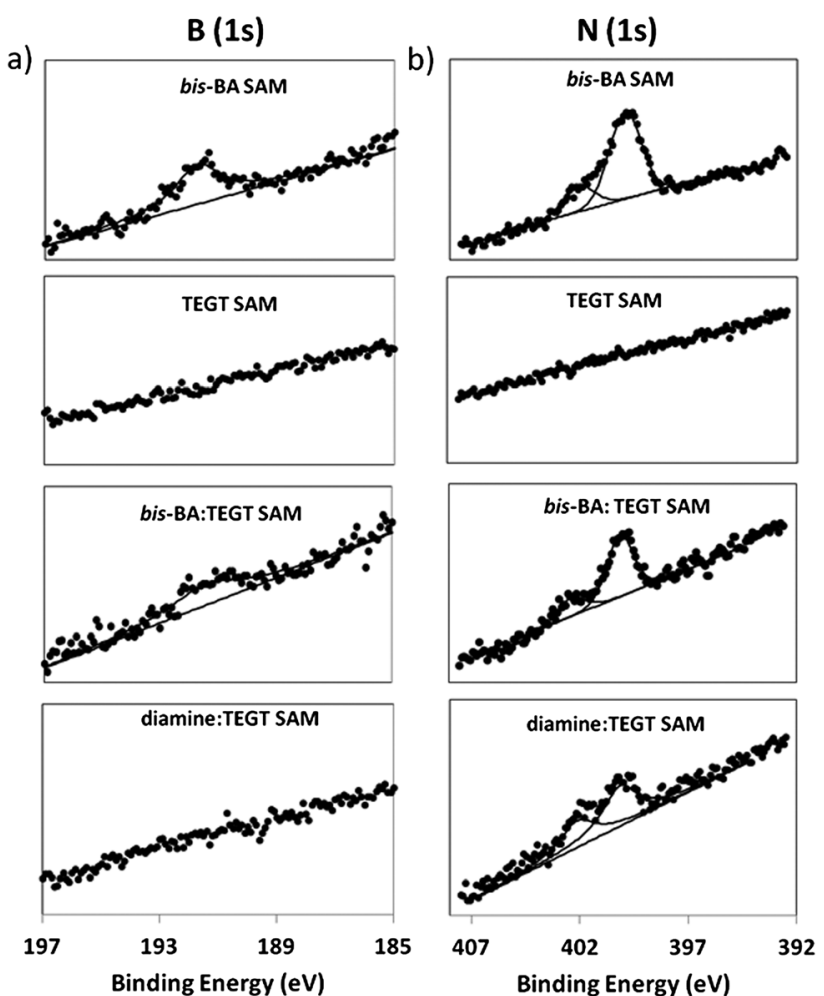

Fig. 2 XPS spectra of (a) B (1s) and (b) N (1s), from, pure bis-BA, pure TEGT, mixed bis-BA : TEGT and mixed diamine : TEGT and SAMs. could facilitate the protonation of the bis-BA and diamine molecules observed.

With the XPS analysis, the ratio of bis-BA : TEGT and diamine : TEGT on the mixed SAM can be further calculated. By integrating the area of the $S(2 p)$ and $N(1 s)$ peaks for the mixed monolayers, a $\mathrm{S}: \mathrm{N}$ ratio of $1: 1$ was obtained. Since both, the bis-BA molecule and the diamine compound, consist of $3 \mathrm{~N}$ atoms and $2 \mathrm{~S}$ atoms and TEGT has no $\mathrm{N}$ and $1 \mathrm{~S}$ atom only, a $\mathrm{S}: \mathrm{N}$ ratio of $1: 1$ corresponds to a ratio of $1: 1$ of bis-BA : TEGT and $1: 1$ of diamine: TEGT on the mixed SAM. Thus, the surface ratio determined by XPS is in close agreement to that determined using the Cassie equation.

\section{Detection of saccharide binding via SPR}

Following the characterisation of the modified-gold surfaces, attention was turned to the investigation of the saccharide binding ability of the 1 : 1 bis-BA : TEGT SAMs-based sensors. Measurements of the interaction between either D-glucose, D-galactose, D-fructose or D-mannose and 1:1 bis-BA: TEGT SAMs was performed by SPR. Different concentrations of saccharides (0.6 mM, $1.25 \mathrm{mM}, 2.5 \mathrm{mM}, 5 \mathrm{mM}, 10 \mathrm{mM}$ and $20 \mathrm{mM}$ ) in PBS solution were used. The concentration range of saccharides was chosen to reflect the range of blood glucose concentrations observed in healthy and diabetic patients; while the definition of a clear diagnostic cut off has proven to be divisive within the medical community, healthy adults would be expected to have a blood glucose level of around $4 \mathrm{mM}$, while diabetes is commonly diagnosed when blood glucose levels are raised above $7 \mathrm{mM}^{26}$

Each saccharide solution was injected over a mixed bisBA : TEGT surface for 5 min to reach equilibrium, followed by a dissociation phase with only PBS buffer flowing over the chip (Fig. 3). It should be noted that for each saccharide all five curves presented in Fig. 3 were performed using the same SAM surface. After the dissociation phase for $2 \mathrm{~min}$, the chip was regenerated for 2 min with an acidified $(\mathrm{pH}=5) 3: 1(\mathrm{v} / \mathrm{v})$
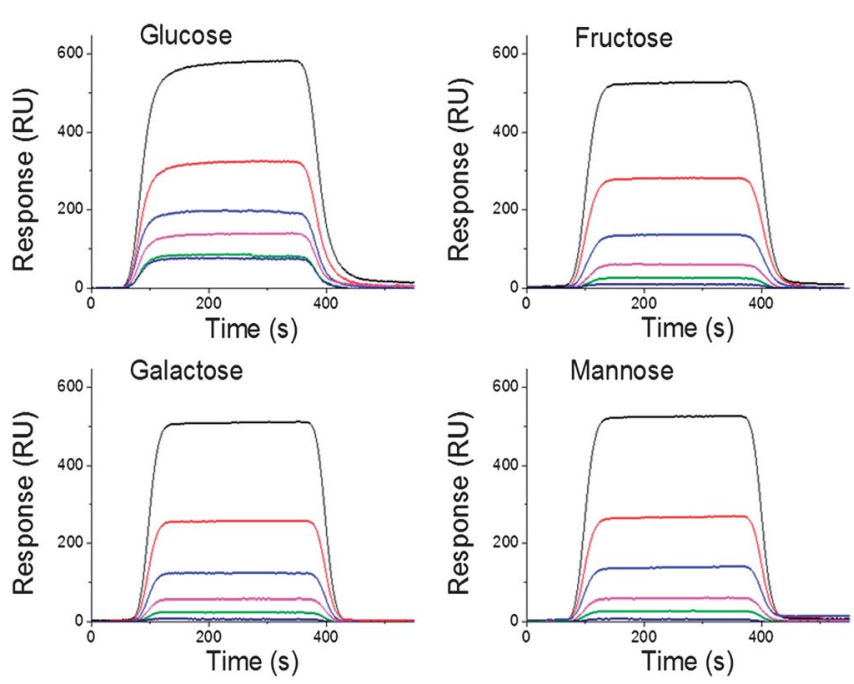

Fig. 3 SPR kinetic measurements showing the binding of D-glucose, D-galactose, D-fructose and D-mannose to $1: 1$ bis-BA : TEGT SAMs using different saccharides concentrations (0.6 mM, $1.25 \mathrm{mM}, 2.5 \mathrm{mM}, 5 \mathrm{mM}, 10 \mathrm{mM}$ and $20 \mathrm{mM})$. 
ethanol : PBS solution to ensure that all bound saccharide was removed from the surface. Regeneration was verified by a return to the baseline established prior to each run.

When considering the SPR data, all hexose sugars exhibited clear concentration dependent-responses, although the intensities differed among the individual sugars. Across all concentrations, glucose produced the largest change in SPR response. To derive affinity binding constants for the interaction between the immobilised boronic acid moieties and the different saccharides in solution, equilibrium analysis were chosen because they can avoid problems resulting from mass transport limitations. ${ }^{27}$ In order to correct for bulk refractive index contributions arising from the differing buffer composition and some possible nonspecific binding to the bis-BA : TEGT SAMs, SPR responses from the control mixed diamine : TEGT were subtracted from those obtained from the bis-BA : TEGT SAMs. The corrected SPR responses at equilibrium $\left(R_{\text {eq }}\right)$ were plotted against the concentration of injected saccharide (CS) (Fig. 4) and fitted to a 1:1 steady-state affinity model. The model utilises a nonlinear least-squares regression method to fit data to the Langmuir adsorption isotherm (eqn (2)). $K_{\mathrm{D}}$ is the dissociation constant of the BA-sugar complex and $R_{\max }$ is the maximum response if all available BA binding sites are occupied. The calculated $K_{\mathrm{D}}$ were inversed, to give the association constant, $K_{\mathrm{A}}$, to allow comparison with data obtained previously from solution. ${ }^{\mathbf{1 2}}$ These values are presented in Table 2.

$$
R_{\mathrm{eq}}=\left(\frac{C_{\mathrm{s}}}{C_{\mathrm{s}}+K_{\mathrm{D}}}\right) R_{\max }
$$

The $K_{\mathrm{A}}$ results illustrate that the surfaces exhibit a higher affinity for glucose, with a comparatively reduced affinity to other hexose sugar isomers, including demonstrating over double the affinity for glucose when compared to fructose (Table 2). These results are comparable with stability constants $\left(K_{\mathrm{OBS}}\right)$ previously observed in solution, producing the same orders of saccharide binding affinities as previously determined. While the absolute values differ this is likely to be caused by the different steric constraints imposed by the surface attachment.

Glucose affinity of the surface is comparable to some biological glucose ligands which have been previously investigated;

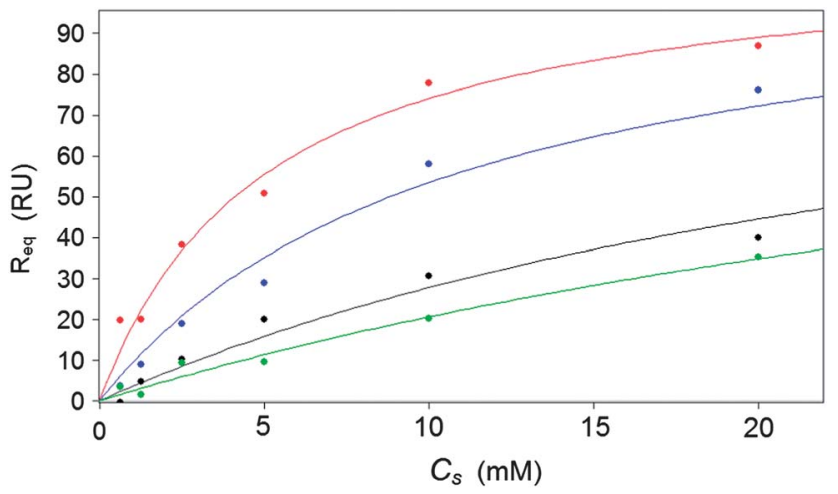

Fig. 4 Calibration curve of control subtracted SPR response change for bis-BA sensor versus glucose (red), fructose (blue), galactose (black) and mannose (green) (0.3 mM, 0.6 mM, $1.25 \mathrm{mM}, 2.5 \mathrm{mM}, 5 \mathrm{mM}, 10 \mathrm{mM}$ and $20 \mathrm{mM}$ ).
Table 2 Table showing calculated $K_{D}$ values for each saccharide. Solution stability constants, $K_{\mathrm{OBS}}$ are included for reference

\begin{tabular}{lcc}
\hline Saccharide & Surface $K_{\mathrm{A}}\left(\mathrm{M}^{-1}\right)$ & Solution $K_{\mathrm{OBS}}{ }^{12}\left(\mathrm{M}^{-1}\right)$ \\
\hline D-Glucose & $203.25 \pm 0.1^{a}$ & 962 \\
D-Fructose & $95.2 \pm 0.1$ & 784 \\
D-Galactose & $33.9 \pm 0.4$ & 657 \\
D-Mannose & $22.9 \pm 0.7$ & 74 \\
${ }^{a}$ Values are standard deviations (S.D.). & \\
\hline
\end{tabular}

bacterial binding proteins utilised in an SPR based sensor have been found to have similar affinities for glucose. ${ }^{28}$ Furthermore, the sensor produced here displays a sensitivity range which is useful to a clinical setting, unlike previously described sensors which have been only useful over much lower saccharide concentrations before the surface becomes saturated.

The results are also in the form of a calibration curve (Fig. 4), exhibiting a detection range over the clinically relevant concentrations of saccharides analysed. Although a response from the other hexose isomers is observed, the impact on glucose measurements in clinical samples would be minimal as glucose is by far the most prevalent saccharide found in blood and other bodily fluids; typically found in concentrations orders of magnitude greater than other saccharides. ${ }^{3}$

\section{Conclusions}

In summary, we have demonstrated, for the first time, that through the marriage of supramolecular design and surface modification the fabrication of a glucose selective sensor surface. Utilising an SPR detector system, the fabricated sensor is able to operate at clinically relevant saccharide concentrations. This offers a range of opportunities for the production of flow past sensors and also the utilisation in miniaturised systems.

\section{Acknowledgements}

P.M.M. acknowledges the Leverhulme Trust (F/00094/BD) and BBSRC (BB/F017618/1) for financial support and the University of Leeds EPSRC Nanoscience and Nanotechnology Facility (LENNF) for access to the XPS. A.S.-B, P.I., J.A.P., J.S.F. and P.M.M. thank the University of Birmingham and Birmingham Science City: Innovative Uses for Advanced Materials in the Modern World (West Midlands Centre for Advanced Materials Project 2), supported by Advantage West Midlands (AWM) and part funded by the European Regional Development Fund (ERDF). J.S.F. and H.-C.W. acknowledge the EPSRC First Grant Scheme (EP/J003220/1). T.D.J. and J.S.F. recognise support from the DTi and ESRC (DT/F00267X/1). Y.-T.L. thanks East China University of Science and Technology and is supported by the Program for Professor of Special Appointment (Eastern Scholar) at Shanghai Institutions of Higher Learning, Shanghai Fundamental Research Project (12JC1403500) and the Sino-UK Higher Education Research Partnership for PhD Studies. Y.-T.L., J.S.F. and T.D.J thank the Catalysis and Sensing for our Environment 
(CASE) network. J.S.F. and T.D.J thank ECUST for visiting professorships. T.D.J thanks the University of Bath. T.D.J. and J.S.F. also thank the Royal Society for support.

\section{References}

1 S. I. Taylor, Cell, 1999, 97, 9-12.

2 E. Mizrachi, S. D. Mansfield and A. A. Myburg, New Phytol., 2012, 194, 54-62.

3 K. M. Manesh, P. Santhosh, A. Gopalan and K.-P. Lee, Anal. Biochem., 2007, 360, 189-195.

4 S. Wild, G. Roglic, A. Green, R. Sicree and H. King, Diabetes Care, 2004, 27, 1047-1053.

5 D. K. Scrafton, J. E. Taylor, M. F. Mahon, J. S. Fossey and T. D. James, J. Org. Chem., 2008, 73, 2871-2874.

6 X. Kang, J. Wang, H. Wu, I. A. Aksay, J. Liu and Y. Lin, Biosens. Bioelectron., 2009, 25, 901-905.

7 T. D. James, M. D. Phillips and S. Shinkai, Boronic Acids in Saccharide Recognition, The Royal Society of Chemistry, Cambridge, 2007.

8 (a) J. H. Holtz and S. A. Asher, Nature, 1997, 389, 829-832; (b) V. L. Alexeev, A. C. Sharma, A. V. Goponenko, S. Das, I. K. Lednev, C. S. Wilcox, D. N. Finegold and S. A. Asher, Anal. Chem., 2003, 75, 2316-2323.

9 D.-H. Yang, N. Takahara, S.-W. Lee and T. Kunitake, Sens. Actuators, B, 2008, 130, 379-385.

10 S. Park, H. Boo and T. D. Chung, Anal. Chim. Acta, 2006, 556, 46-57.

11 (a) J. S. Fossey, F. D'Hooge, J. M. van den Elsen, M. P. Pereira Morais, S. I. Pascu, S. D. Bull, F. Marken, A. T. Jenkins, Y. B. Jiang and T. D. James, Chem. Rec., 2012, 12, 464-478; (b) S. A. Elfeky, F. D'Hooge, L. Poncel, W. Chen, S. P. Perera, J. M. H. van den Elsen, T. D. James, A. T. A. Jenkins, P. J. Cameron and J. S. Fossey, New J. Chem., 2009, 33, 1466; (c) J. S. Fossey and T. D. James, in Reviews in Fluorescence 2007, ed. C. D. Geddes, Springer New York, New York, NY, 2009, vol. 2007, pp. 103-118; (d) R. Nishiyabu, Y. Kubo, T. D. James and J. S. Fossey, Chem. Commun., 2011, 47, 11241150; (e) R. Nishiyabu, Y. Kubo, T. D. James and J. S. Fossey, Chem. Commun., 2011, 47, 1106-1123; $(f)$ T. A. Schuller, M. Kuball, S. E. Flower, T. D. James, J. S. Fossey, D. Marcon, J. Das, S. Degroot, M. Germain and A. Sarua, Sens. Actuators, B, 2011, 160, 1078-1081; (g) Y.-J. Huang, W.-J. Ouyang, X. Wu, Z. Li, J. S. Fossey, T. D. James and Y.-B. Jiang, J. Am. Chem. Soc., 2013, 135, 1700-1703; (h) X. Wu, Z. Li, X.-X. Chen, J. S. Fossey, T. D. James and Y.-B. Jiang, Chem. Soc. Rev., 2013, 42, 8032-8048.

12 S. Arimori, M. L. Bell, C. S. Oh, K. a. Frimat and T. D. James, J. Chem. Soc., Perkin Trans. 1, 2002, 803-808.
13 (a) M. Lee, T.-I. Kim, K.-H. Kim, J.-H. Kim, M.-S. Choi, H.-J. Choi and K. Koh, Anal. Biochem., 2002, 310, 163-170; (b) E. Shoji and M. S. Freund, J. Am. Chem. Soc., 2002, 124, 12486-12493; (c) D. G. Hall, Boronic acids, Wiley-VCH, Weinheim, 2005; (d) F. D'Hooge, S. A. Elfeky, S. E. Flower, S. I. Pascu, A. T. A. Jenkins, J. M. H. V. D. Elsen, T. D. James and J. S. Fossey, RSC Adv., 2012, 2, 3274.

14 C. D. Bain, E. B. Troughton, Y. T. Tao, J. Evall, G. M. Whitesides and R. G. Nuzzo, J. Am. Chem. Soc., 1989, 111, 321-335.

15 H.-C. Wang, H. Zhou, B. Chen, P. M. Mendes, J. S. Fossey, T. D. James and Y.-T. Long, Analyst, 2013, DOI: 10.1039/ c3an01234d.

16 (a) C. L. Yeung, P. Iqbal, M. Allan, M. Lashkor, J. A. Preece and P. M. Mendes, Adv. Funct. Mater., 2010, 20, 2657-2663; (b) C.-P. Chak, L.-H. Chau, S.-Y. Wu, H.-P. Ho, W. J. Li, P. M. Mendes and K. C.-F. Leung, J. Mater. Chem., 2011, 21, 8317-8323; (c) A. Pranzetti, S. Salaün, S. Mieszkin, M. E. Callow, J. A. Callow, J. A. Preece and P. M. Mendes, Adv. Funct. Mater., 2012, 22, 3672-3681.

17 (a) A. Hemmi, T. Imato, Y. Aoki, M. Sato and N. Soh, Sens. Actuators, B, 2005, 108, 893-898; (b) J. R. Son, G. Kim, A. Kothapalli, M. T. Morgan and D. Ess, J. Phys.: Conf. Ser., 2007, 61, 1086.

18 L. Li, S. Chen, J. Zheng, B. D. Ratner and S. Jiang, J. Phys. Chem. B, 2005, 109, 2934-2941.

19 S. Flink, F. Van Veggel and D. N. Reinhoudt, J. Phys. Org. Chem., 2001, 14, 407-415.

20 (a) A. Ulman, Chem. Rev., 1996, 96, 1533-1554; (b) H. L. Zhang, J. Zhang, H. Y. Li, Z. F. Liu and H. L. Li, Mater. Sci. Eng., C, 1999, 8-9, 179-185.

21 (a) C. D. Bain, H. A. Biebuyck and G. M. Whitesides, Langmuir, 1989, 5, 723-727; (b) C.-H. Shen and J.-C. Lin, Langmuir, 2011, 27, 7091-7098.

22 A. B. D. Cassie, Discuss. Faraday Soc., 1948, 3, 11-16.

23 R. I. Carey, J. P. Folkers and G. M. Whitesides, Langmuir, 1994, 10, 2228-2234.

24 P. M. Mendes, K. L. Christman, P. Parthasarathy, E. Schopf, J. Ouyang, Y. Yang, J. A. Preece, H. D. Maynard, Y. Chen and J. F. Stoddart, Bioconjugate Chem., 2007, 18, 1919-1923.

25 F. Sancenón, A. B. Descalzo, J. M. Lloris, R. Martínez-Máñez, T. Pardo, M. a. J. Seguí and J. Soto, Polyhedron, 2002, 21, 1397-1404.

26 I. F. Godsland, J. A. Jeffs and D. G. Johnston, Diabetologia, 2004, 47, 1157-1166.

27 P. Schuck, Annu. Rev. Biophys. Biomol. Struct., 1997, 26, 541566.

28 H. V. Hsieh, Z. A. Pfeiffer, T. J. Amiss, D. B. Sherman and J. B. Pitner, Biosens. Bioelectron., 2004, 19, 653-660. 